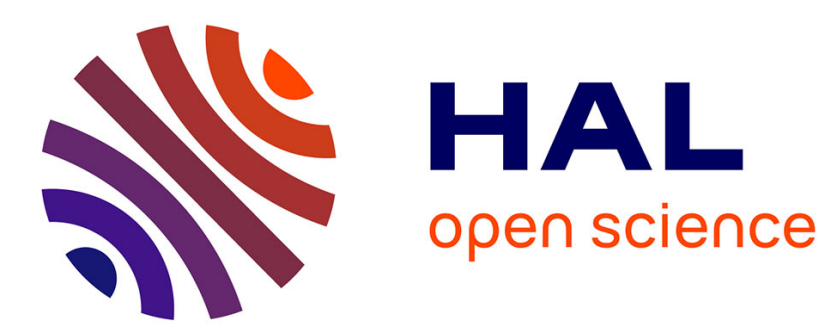

\title{
RESSOURCES ET CROISSANCE DANS LE MONDE ANGLO-NORMAND: SOURCES ET HYPOTHĖSES
}

\author{
Mathieu Arnoux
}

\section{To cite this version:}

Mathieu Arnoux. RESSOURCES ET CROISSANCE DANS LE MONDE ANGLO-NORMAND: SOURCES ET HYPOTHĖSES. Anglo-Norman Studies XL, Boydell and Brewer Limited, pp.53-62, 2018, Proceedings of the Battle Conference 2017, 10.1017/9781787443051.004 . hal-03124954

\section{HAL Id: hal-03124954 \\ https://hal-univ-paris.archives-ouvertes.fr/hal-03124954}

Submitted on 10 Feb 2021

HAL is a multi-disciplinary open access archive for the deposit and dissemination of scientific research documents, whether they are published or not. The documents may come from teaching and research institutions in France or abroad, or from public or private research centers.
L'archive ouverte pluridisciplinaire HAL, est destinée au dépôt et à la diffusion de documents scientifiques de niveau recherche, publiés ou non, émanant des établissements d'enseignement et de recherche français ou étrangers, des laboratoires publics ou privés. 


\title{
RESSOURCES ET CROISSANCE DANS LE MONDE ANGLO-NORMAND: SOURCES ET HYPOTHÈSES
}

\author{
Mathieu Arnoux
}

Resource has always been a missing element in medieval economic history. According to the implicitly Malthusian model, which underlies most of the current literature, narrative about growth and crisis does not need a chapter on natural resources: where there is economic and demographic growth there must be resources; when crisis happens, resources must be insufficient. Such an interpretation takes its roots in the theses of Thomas Malthus (1766-1834) and Jean-Baptist Say (1767-1832), two major economists of early industrial times. Instead, in pre-industrial economies environment is not considered an opportunity for individual profit or entrepreneurial purpose: the main issue is legitimate needs and livelihood. Any thorough analysis of medieval economics should start with people's needs and the question of allowing them a means of subsistence, rather than with an assessment of potential benefits. The main challenge for the historian is to find useful evidence. For the Central Middle Ages, Fritz Curschman's book Hungersnöte in Mittelalter (1901) provides a very useful collection of cases, which, for the eleventh and twelfth centuries, casts light on the process of some severe famines, most of them in Flanders and Rhineland cities. The Anglo-Norman realm seems to have suffered less shortage of livelihood than the German regions. The abundance of harvests around the Channel shores is not easy to describe, still less to explain. For England, an enormous amount of literature, about manorial economy and open field husbandry, suggests that royal power and seigniorial institutions, which shaped the agrarian landscape, were the organizers of the allocation of wealth and the arbiters of need. Despite its pervasive influence on English political evolution, Normandy was a very different case. Medieval historiography and charters indicate that local markets were the real centres of the economic and social system. The many settlements of tithes conflicts in the late twelfth century and many more grain annuity contracts for the thirteenth century provide evidence of a complex system, whose many components all mattered: the nature of the crops (wheat, rye, oats and barley), the social identity of the consumers, the market and credit organization, pricing and assessment of values. This paper argues that any resource system could be very different according to place, but that it was always the core of any social organization.

La question des ressources reste un point obscur dans les recherches sur l'histoire économique du Moyen Âge. ${ }^{1}$ Le plus souvent, la constatation d'une phase de croissance économique et démographique suffit à laisser préjuger de l'existence des ressources mises en œuvre dans ce processus et l'observation d'une crise incite à mettre en question leur insuffisance. Derrière ce raisonnement simple se dissimule l'application mécanique d'un argument bien connu, l'hypothèse malthusienne, qui lie

1 On trouvera dans cet article les hypothèses préalables et les premiers résultats d'une enquête en cours sur les systèmes de ressources dans le monde médiéval. D'où son aspect inachevé ou, pour mieux dire, expérimental. 
croissance et décroissance au caractère limité des ressources disponibles. L'idée en est simple et a pour elle une apparence d'évidence: l'existence d'une ressource vitale permet la mise en place d'un processus de croissance; l'insuffisance ou la disparition de cette ressource en détermine l'arrêt. ${ }^{2}$ Ceux qui ont recours à ce raisonnement n'observent pas le plus souvent qu'il constitue en fait un modèle comportemental de type biologique, adapté à l'observation du cycle des espèces dans un écosystème clos, appartenant à ce que les biologistes appellent un équilibre proie-prédateur. Dans sa forme simple, il ne prévoit pas que les populations observées puissent changer leur rapport aux ressources, c'est-à-dire leur mode de consommation. La référence à Thomas Malthus (1766-1834), présente chez la plupart de ceux qui le mettent en œuvre, doit inciter à regarder de plus près la genèse de ce modèle, lié aux débats des économistes sur la révolution industrielle britannique.

Le mot 'ressource', utilisé le plus souvent dans ce raisonnement pour désigner les richesses naturelles impliquées dans le processus de croissance comme dans les crises, renvoie au même contexte. Tant en français qu'en anglais, il a jusqu'au début du dix-neuvième siècle un sens tout à fait différent, comme en témoigne l'entrée que lui réserve le dictionnaire de Samuel Johnson, identique à celle des dictionnaires français pour le même vocable: Resource: [ressource, Fr.] Some new or unexpected means that offer; resort; expedient. ${ }^{3}$ Une ressource est donc quelque chose qui permet de se tirer d'un mauvais pas, et non l'ensemble de ce qui supporte une vie prévisible. En poursuivant l'enquête dans les lexiques et bases de données de l'ancien français (ressource/ressort), de l'anglo-normand (resource/resort) et du moyen anglais (resort) le sens du mot se précise, et en particulier sa fidélité à la racine latine resurgere, 'ressusciter' ou 'resurgir', qui s'exprime de manière frappante dans le sens 'action de s'envoler' (d'un oiseau de proie, en particulier). ${ }^{4}$

C'est un autre économiste, le Français Jean-Baptiste Say (1767-1832), qui est à l'origine du nouveau sens du mot, celui de richesse naturelle disponible. Le raisonnement s'inscrit dans les premières pages de son Traité d'économie politique, où son analyse des 'agents naturels' donne naissance à la notion de richesses virtuellement inépuisables et dépourvues de valeur monétaire, et donc de prix, dont la mise en œuvre permet la croissance de la richesse des hommes. Le mot 'ressources', absent de la première édition (1803), s'impose dans les éditions suivantes (1816-1826) et devient dès lors un concept-clé de la pensée économique. Ce changement de sens d'un mot communément employé n'apparaît pas aisément dans l'usage qui est en fait pas la suite: l'utilité du nouveau concept pour décrire les réalités contemporaines lui confère un aspect d'évidence. Mais il est porteur d'anachronisme quand il est utilisé pour analyser les situations anciennes.

De fait, dans les sociétés préindustrielles en général, et dans celles du Moyen Âge en particulier, on ne trouve pas l'idée qu'existe dans la nature une richesse virtuellement illimitée, disponible pour l'action productive ou entrepreneuriale des individus. La notion même d'une masse de biens répartis dans l'environnement des

\footnotetext{
2 Présentation claire et nuancée de cet instrument analytique dans J. Hatcher et M. Bailey, Modelling the Middle Ages. The history and theory of England's Economic Development, Cambridge 2001; le livre de Gregory Clark, A farewell to the Alms: a Brief Economic History of the World, Princeton 2009, montre comment un usage sans nuance de l'argument malthusien peut transformer un récit à prétention historique en pure idéologie.

3 Cette entrée restera inchangée depuis la première édition (1755) jusqu'à l'édition posthume de 1828 .

4 Godefroy, Dictionnaire de l'Ancienne Langue Française, 10 vols, Paris 1881-1902, VII, 106 : 'En marches et en la riviere/en ces estangs et en ces sources/ La faisoit de si belles resources/Qu'ung falcon sans cloches, sauvaiges/Ne fist oncques tel vasselaige (Gacé de La Bigne, Deduiz)' ; le terme a conservé ce sens dans le langage aéronautique.
} 
sociétés, qu'il convient de répartir en la valorisant ne saurait en aucun cas décrire l'organisation de ces sociétés, où les acteurs s'interrogent plutôt sur les besoins légitimes et utiles à satisfaire que sur les richesses à allouer. S'il convient de rechercher des mots d'ancien français pour exprimer ces notions, il faudrait alors parler de necessité, de besoin et de substance (nede, substaunce ou levelihede en moyen anglais). Si l'on veut restituer sa lisibilité à l'organisation des économies médiévales, il faut donc s'interroger sur les processus de légitimation des besoins des individus et sur les institutions et organisations créées pour assurer leur satisfaction par un usage légitime des richesses de la création. Dans cette perspective, le thème évangélique des 'œuvres de miséricorde', notion que l'ancien français regroupe sous la notion générale de merci (moyen anglais mercie), doit être considéré comme un programme économique minimal (nourrir les affamés, vêtir ceux qui sont nus, héberger les sans-abris) autant que comme une injonction spirituelle renvoyant à la notion de charité. ${ }^{5}$

\section{Disettes et famines comme marqueurs de la nécessité}

S'il permet de construire un questionnaire plus cohérent avec les catégories médiévales, ce déplacement de notre enquête depuis la notion contemporaine de ressources vers celle ancienne de subsistance implique la recherche d'informations permettant d'éclairer de façon concrète un pan de l'existence social mieux éclairé le plus souvent par des écrit prescriptifs que par des sources narratives ou normatives. L'ouvrage classique de Fritz Curschmann sur les famines dans l'Europe du haut moyen âge (1900) offre un point de départ précieux, en particulier grâce aux annexes où l'historien a rassemblé une large part des mentions annalistiques, historiographiques ou hagiographiques relatives aux crises frumentaires européennes jusqu'au XIII' siècle. $^{6}$

Pour l'essentiel, ces mentions se limitent à des expressions figées et limitées: fames valida ('grave'famine) ou mortalitas maxima ('très grande mortalité') le plus souvent. L'enquête de Curschmann met pourtant en lumière l'existence pour les régions rhénanes et flamandes aux onzième et douzième siècles d'une série impressionnante de descriptions de crises frumentaires insérée dans des recueils d'actes abbatiaux ou épiscopaux et de vitae d'ecclésiastiques ou de princes. Dans chaque cas, la description d'une situation particulièrement désastreuse permet de mettre en évidence la capacité de l'homme d'église ou du puissant à servir le groupe de fidèles dont la protection lui a été dévolue. Très cohérents dans leur argumentation, ces textes, qui évoquent souvent des personnages apparentés, permettent d'esquisser une typologie des situations de crises. Si une météorologie inclémente constitue le plus souvent la cause immédiate, les récits du processus sont révélateurs d'une hiérarchie implicite des périls et des réactions qu'ils doivent susciter chez les gouvernants. Un premier niveau de gravité est constitué par l'insuffisance strictement locale des récoltes, qui appelle la distribution des réserves conservées dans les granges de l'institution. Quand ces ressources ne suffisent pas, il faut recourir aux moyens monétaires, qui sont mis en œuvre sur les marchés locaux ou régionaux pour acquérir des grains. Après épuisement des monnaies, les vases et objets précieux de l'institution sont vendus, ou plus probablement mis en gage. Dans les

5 Les réflexions qui suivent prennent leur origine dans G. Todeschini, Les Marchands et le temple. Le cercle vertueux de la richesse dans l'Europe médiévale et moderne, Paris 2017.

6 F. Curschmann, Hungersnöte in Mittelalter. Ein Beitrag zur Deutschen Wirtschaftsgeschichte des 8 bis 13 Jahrhunderts, Leipzig 1900, 89 et suivantes. 
cas les plus graves, lorsque la récolte ne suffit pas, que les réserves sont épuisées et que les marchés font défaut, la question de l'alimentation se pose dans l'urgence et il faudra des qualités exceptionnelles à l'homme de Dieu pour faire face à la situation.

Pour l'essentiel, les textes rassemblés par Curschmann concernent une aire limitée à la Flandre et aux régions rhénanes. Il s'agit d'un espace démographiquement et économiquement dynamique, caractérisé dès avant l'an mil par un fort mouvement d'urbanisation et par une implication forte des institutions religieuses dans le gouvernement des communautés. La fréquence relative des crises alimentaires graves durant cette période de croissance attire l'attention sur la complexité et l'instabilité, dans cette région, des relations entre villes et espaces ruraux. Le problème, nous disent les mêmes sources, n'est pas seulement économique et alimentaire, mais aussi politique et religieux : ce qui est en jeu durant les onzième et douzième siècles est à la fois la domination des pouvoirs urbains sur les espace ruraux et l'affirmation des parties les plus dynamiques des sociétés urbaines face aux dirigeants issus de l'aristocratie carolingienne. Dans une perspective plus large, ce mouvement de recomposition politique et économique concerne à la fois les régions rhénanes et flamandes et, à l'autre extrémité de l'Empire, celles de l'Italie centro-septentrionale.

L'application du questionnaire de Curschmann aux sources anglo-normandes ne fait pas apparaître de textes qu'il aurait négligés et qui permettraient d'étendre ses hypothèses sur l'importance de la question frumentaire à l'extrémité occidentale du continent. Les récits de famines sont peu fréquents dans cet espace et ne peuvent se lire dans la même perspective. On en trouve un exemple paradoxal dans l'Inventio et miracula sancti Wulfranni, recueil écrit à Saint-Wandrille au milieu du XIe siècle, dont un passage est relatif à une grande famine, survenue dans les années 1030, peut-être la même que celle à laquelle Raoul Glaber avait consacré un récit célèbre. ${ }^{7}$ À la différence du récit laissé par Raoul et de ceux rassemblés par Curschmann, qui frappent par leur précision et l'abondance des détails concrets et situés, le récit fait par un moine normand est rhétorique, imprécis et comme de seconde main. Tout se passe comme si, une génération plus tard à peine, l'événement n'avait pas laissé de souvenir direct dans le duché. ${ }^{8}$

De fait, une enquête plus approfondie sur les épisodes de famine évoqués dans les textes des historiens normands et anglo-normands, Guillaume de Jumièges, William de Malmesbury et Orderic Vital, montre que celle-ci ne résulte pas le plus souvent d'une insuffisance des récoltes par suite d'un dérèglement climatique. Elle apparaît le plus souvent comme dégât collatéral d'une conjoncture politique troublée : c'est le cas pour la croisade, chez Orderic Vital, ou pour les troubles de la succession de Guillaume le Conquérant chez William de Malmesbury. C'est bien évidemment le cas pour la dévastation du Yorkshire (the harrying of the North) en 1069, dont la plupart des récits soulignent qu'elle implique la responsabilité morale du roi Guillaume. ${ }^{9}$ Par ailleurs, les villes et leurs habitants ne sont pas des acteurs de premier plan dans ces récits et leur approvisionnement n'y constitue pas un enjeu majeur. Sources rhénanes-flamandes et anglo-normandes renvoient à un

7 Raoul Glaber, Histoires, IV, c. 13, trad. M. Arnoux, Turnhout, 1995, 247; Rodulfus Glaber, Opera, éd. et trad. John France, Neithard Bulst et Paul Reynolds, OMT, Oxford 1989, 190-3.

$8 \quad$ Inventio and Miracula Sancti Vulfranni, éd. Dom J. Laporte, Rouen-Paris 1938, Mélanges publié par la Société de 1'Histoire de Normandie, 14e série, 53-5; E. M. C. van Houts, 'Historiography and Hagiography at Saint-Wandrille: the Inventio and Miracula Sancti Vulfranni', ANS 12, 1990, 233-51.

9 Ce thème fait l'objet d'un article particulier en cours de rédaction; sur le Harrying of the North, cf. D. Bates, William the Conqueror, New Haven-London 2016, 313-21. 
processus différent d'urbanisation : les famines de Gand, Lièges ou Trêves trouvent leur origine dans une croissance urbaine précoce, mettant en tension les ressources agraires régionales. De ce point de vue, l'urbanisation plus lente, et moins massive à terme, qui caractérise le monde anglo-normand, permet une plus grande sureté d'approvisionnement des centres urbains. Dans cet espace équilibré, les crises graves résultent plutôt de disfonctionnement politiques.

\section{Sources et modèles économiques et de l'abondance}

Ce n'est donc pas dans les sources relatives aux crises de subsistance qu'il faut aller chercher des informations sur la question des ressources dans le monde anglonormand, qui paraît, au moins jusqu'à la fin du treizième siècle, jouir d'une bonne sécurité dans l'approvisionnement. De fait, pour le duché comme dans le royaume, la tradition littéraire loue la fertilité des champs et l'abondance des récoltes, qu'il s'agisse du prologue de l'Historia Anglorum d'Henri de Huntingdon ou des éloges faits à la Neustrie puis à la Normandie par Wace et Benoît de Sainte-Maure ${ }^{10}$. Ce topos est repris dans les descriptions de provinces que Barthélemy l'Anglais a insérées dans son encyclopédie des Propriétés des choses : en Angleterre « La terre y est tres bonne et able a porter divers fruictz » et « Normandie est un grant pays et habondant en bledz, en boys en prés et en bestes sauvaiges et privees $\gg .{ }^{11}$ La céréaliculture offre donc une voie d'accès au problème des ressources dont les blés constituent à l'évidence une part centrale et essentielle pour les deux rives de la Manche. Une exploration globale de ce thème, même superficielle, excèderait largement l'objet de cet article, et ne permettrait sans doute pas de répondre aux questions qu'il soulève. Elle impliquerait en particulier de s'interroger sur les liens que le modèle social des trois ordres, qui connut dans le monde anglo-normand son plus grand développement, entretient avec cette situation de suffisance, sinon d'abondance. Dans les lignes qui suivent, j'esquisserai une comparaison entre Normandie et l'Angleterre en me fondant sur la bibliographie consacrée au royaume, pour mettre en évidence le cas très différent offert par le duché.

Il n'est peut-être pas nécessaire d'insister sur l'extraordinaire importance de la production céréalière dans la construction sociale, politique et économique de l'Angleterre, bien au-delà du Moyen Âge. Pour la période qui nous intéresse, les céréales sont au cœur de deux thèmes historiographiques essentiels, auxquels a été consacrée une littérature proprement gigantesque, la question de la seigneurie manoriale d'une part, celle de la géographie agraire et des paysages d'open-field d'autre part. ${ }^{12} \mathrm{Il}$ convient ici d'insister à nouveau sur l'extrême originalité de la situation anglaise dans les deux cas, en particulier du point de vue des sources mise à disposition des historiens : il n'existe rien sur le continent, et en particulier en Normandie,

10 Henry, Archdeacon of Huntingdon, Historia Anglorum, éd. D. Greenway, OMT, Oxford 1996, 1-2 ; M. Arnoux, Le temps des laboureurs. Travail, ordre social et croissance en Europe (xie-xive siècle), Paris 2012, 123-5.

11 Le proprietaire des choses, traduction par Jean Corbechon (1372) du De proprietatibus rerum, éd. Lyon 1495 (non paginée), XV, c. 106 (est autem gleba eius frugifera et pinguis campis, nemoribus et pratis insignis) et 14 (gleba optima et diuesis fructibus ualde apta; ibi oues lanigere in precipua abundantia); la notice consacrée à la Flandre (XV, c. 38) est très différente: 'Flandre combien que elle soit petite quant au siege, toutesfoys est elle bonne terre et pleine de biens moultz singuliers car elle est pleine de pastures de beufs, de brebis et d'autres bestes'.

12 Reginald Lennard, Rural England, 1086-1135. A study of Social and Agrarian Conditions, Oxford 1959; Bruce M. S. Campbell, English Seigniorial agriculture 1250-1450, Cambridge 2000; Tom Williamson, Shaping Medieval Landscapes. Settlement, Society, Environment, Oxford 2003. 
qui ressemble, même de façon éloignée, aux séries de comptes manoriaux ou aux innombrables sites de villages désertés par suite de leur enclosures. Du point de vue de la compréhension des pratiques des acteurs, il n'existe rien de comparable aux multiples traités de husbandry des treizième et quatorzième siècles. ${ }^{13}$

Souvent considérés comme des éléments disjoints renvoyant chacun à des dynamiques différentes et à une chronologie particulière, ces divers éléments composent de notre point de vue un ensemble cohérent, auquel il est de surcroît possible d'approcher par une histoire intellectuelle. On ne souligne pas le plus souvent que l'extraordinaire (et rarissime) croquis de charrue inséré avec sa légende bilingue, vers 1280 dans le cartulaire du couvent des moniales cisterciennes de Nun Coton (Lincs.) ${ }^{14}$ renvoie à la fois à la tradition d'agronomie domaniale de Walter de Henley, mais aussi à la pratique universitaire des lexiques d'Adam du Petit-Pont et d'Alexandre de Garlande. ${ }^{15}$ Dans la même région, la rédaction par l'évêque de Lincoln Robert Grosseteste d'un traité de gestion domaniale, dont sont conservées plusieurs versions en langues latine ou anglo-normande, doit inciter à s'interroger sur les implications intellectuelles et religieuses d'activités agraires trop souvent envisagées d'un point de vue étroitement matériel et économique. ${ }^{16}$ Dans cette perspective, l'originalité anglaise est d'avoir enraciné traditions et pratiques politiques, religieuses, intellectuelles, sociales et économiques dans la céréaliculture, objet central dans le fonctionnement de la société anglaise. L'apparition, au terme de ce cycle de croissance et dans le contexte de la crise du quatorzième siècle, du personnage littéraire du ploughman, inconnu dans le reste de l'Europe, est un signe de cette centralité de la céréaliculture. ${ }^{17}$ Pour la période précédente, il est remarquable qu'Henri de Huntingdon, dont nous connaissons par son œuvre historique la sensibilité aux questions de ressources, n'ait pas retenu les céréales dans la liste des plantes décrites dans son Hortus Anglicanus : les grains, à la culture desquels les rustici consacrent leur existence, ne sont pas, comme les plantes médicinales, un objet de connaissance naturelle, mais bien plutôt un objet social et politique. ${ }^{18}$

\section{En Normandie: dîmes, rentes, marchés.}

Face à cette construction institutionnelle de la ressource céréalière, dont l'essentiel se met en place au cours du douzième siècle, le duché de Normandie se trouve dans une situation singulière: l'aristocratie ducale et les institutions ecclésiastiques normandes tiennent une place essentielle dans la transformation du royaume, que ce soit par l'exercice sans partage de l'autorité politique et religieuse ou par l'introduction d'un bilinguisme aux conséquences importantes. Durant les douzième et treizième siècles, les manoirs appartenant à des seigneurs laïcs sont, pour une large part dans la main de seigneurs normands ou d'institutions religieuses du duché. Pour autant, il ne semble pas que les innovations découvertes ou intro-

13 Walter of Henley and Other Treatises in Estate Management and Accounting, éd. D. Oschinsky, Oxford 1971.

14 Oxford Bodleian Library, MS Top. Lincs. d. 1, fol. 53.

15 Tony Hunt, Teaching and Learning Latin in 13 $3^{\text {th }}$-century England, Cambridge 1991.

16 Walter of Henley, 388-409: Reules ke le bon eveske de Nichole Robert Grosseteste fist a la comtesse de Nichole de garder et governer terres et hostel ('Règles que le bon évêque de Lincoln Robert Grosseteste composa pour la comtesse de Lincoln pour le gouvernement de ses terres et de son hôtel').

17 Arnoux, Le temps des laboureurs, 173-95.

18 Henry of Huntingdon, Hortus Anglicanus. A Verse Herbal of the Twelfth Century, éd. W. Black, Toronto 2012. 
duites en Angleterre aient fait l'objet d'expérimentation en terre normande : pas de seigneurie manoriale, pas de construction paysagère d'open-field, pas de littérature agronomique. Cette situation pose un double problème à l'historien : rendre compte de la dissymétrie de la construction anglo-normande mais aussi trouver les sources qui permettent de comparer les deux régions. ${ }^{19}$

Les sources du onzième siècle semblent indiquer une certaine maturité de l'économie de marché en Normandie. C'est en particulier le témoignage de Raoul le Glabre, relevant l'étonnante rigueur des pratiques de marché chez les Normands : 'Parmi eux, passait pour voleur ou pillard quiconque, prétendant en affaire plus que le juste prix, ou trompant sur la qualité, s'enrichissait aux dépens d'autrui' ${ }^{20}$ Le texte bien connu de Guillaume de Jumièges relatif à la grande révolte des paysans au début du règne de Richard II peut aussi se lire en ce sens, en ce qui concerne les motivations des révoltés : 'tous les paysans des différents comtés de la patrie normande, décidèrent, au cours d'innombrables conciliabules, de vivre selon leurs volonté, de sorte que tant pour l'usage modéré des forêts que pour la vente des eaux courantes, ils useraient de leurs propres règles sans que les droits anciens leur soient un obstacle. ${ }^{21}$ Sans qu'on puisse en offrir une traduction très précise, on peut interpréter les expressions compendia silvarum et commercia aquarum comme désignant des évaluations économiques des richesses naturelles que sont les forêts et les eaux des rivières, sur lesquelles les paysans se trouvent en conflit avec le groupe seigneurial. Il est par ailleurs notable qu'aucune des expressions ne fasse directement allusion à de la nourriture, ce qui semble indiquer que l'alimentation n'est pas la source du conflit. Il est évidemment difficile d'aller plus loin pour le onzième siècle, en raison de la rareté des informations, mais les sources beaucoup plus abondantes et précises des deux siècles suivants permettent de mieux poser les problèmes. Deux pistes s'offrent pour mener cette enquête, la première relative aux dîmes, la deuxième relative aux rentes.

Tous les historiens du duché savent la place considérable occupée dans les chartriers et cartulaires par les actes relatifs aux dîmes. Cette abondance d'information, qui commence au onzième siècle, m'avait conduit à accorder une signification sociale importante à cette institution, usuellement considérée comme exclusivement ecclésiastique. ${ }^{22}$ De ce point de vue, la ressource exceptionnelle par son ampleur que constitue les dîmes paroissiale doit être considérée comme l'un des enjeux des relations de concurrences ou de coopération qui unissent clercs et laïcs au sein des communautés paroissiales. Dans la perspective d'une enquête sur la question des ressources, les actes relatifs aux dîmes, et en particulier les multiples sentences et lettres décrétales réglant les conflits à leur sujet, offrent un observatoire exceptionnel sur les pratiques agraires. Plus que les actes relatifs aux restitutions de

19 M. Arnoux, 'Border, Trade Route or Market? The Channel and the Medieval European Economy from the Twelfth to the Fifteenth Century', ANS, 36, 2014, 39-52; M. Arnoux, 'Entre Paris et la Manche. Réflexions sur la place de la Normandie dans les espaces anglo-normands et français ( $\mathrm{XII}^{\mathrm{e}}$-XV ${ }^{\mathrm{e}}$ siècles)', dans Penser les mondes normands médiévaux, éd. D. Bates et P. Bauduin, Caen 2016, 493-502.

20 Nempe furi ac predoni apud illos comparabatur quicumque hominum in aliquo negotio plus iusto yel falsum quippiam uenundandum mentiens (Raoul Glaber, Histoires, I, c. 21, p. 75; Rodulfus Glaber, 36-7).

21 GND V, c. 2, II, 8-9: Rustici unanimes per diversos totius Normannicae patriae comitatus plurima agentes conventicula juxta suos libitus vivere decernebant, quatinus tam in silvarum compendiis quam in aquarum commerciis, nullo obsistente ante statuti juris obice legibus uterentur suis. Pour la phrase accentuée la traduction anglaise corrigée est: 'moderated use of the forests as well as the sale of waterways' au lieu de la traduction publiée : 'in respects both of short cuts through the woods and of the traffic of the rivers'.

22 Arnoux, Le temps des laboureurs, 221-55. 
dîmes par leurs détenteurs laïcs, qui témoignent de l'application progressive des principes de la réforme grégorienne, ce sont les actes multiples mettant terme aux conflits né de la possession partagée des dîmes par les communautés paroissiales et les établissements réguliers qui mettent en lumières les caractères originaux des régimes agraires.

Malgré leur précision, ces textes ne traitent pas des conditions concrètes de la production. En particulier, ils ne permettent jamais de décider du caractère biennal ou triennal des rotations, une distinction cruciale pour les agronomes anglais dans leur recherche de la façon optimale d'associer parcelles cultivées et instruments de labour. La description extraordinairement concrète qui est donnée des opérations de moisson dans une sentence des années 1198-1203 relative à la paroisse de Fontenay-le-Marmion, au sud de Caen, énumère les instruments nécessaires à la levée de leurs dîmes par les moines de l'abbaye cistercienne de Barbery et par le seigneur Robert d'Ouffières : trois charrettes attelées avec leurs équipage pour les moines et une pour Robert, ainsi qu'une cinquième charrette commune et un homme avec son cheval allant et venant parmi les champs moissonnés. Dans ce cas, les juges n'hésitent pas à fixer par écrit les moyens nécessaires à la bonne exécution des opérations légales liées à la récolte. ${ }^{23}$

Les juges sont aussi attentifs, presque toujours, à distinguer entre eux les grains récoltés et engrangés : froment, orge avoine. Ce souci de précision est significatif de la destination socialement différenciée des céréales, le froment revenant aux ecclésiastiques, curés, chanoines ou moines et le méteil, le seigle, l'avoine et l'orge étant destinés aux églises paroissiales. Les historiens de l'alimentation ont depuis longtemps montré que le prestige reconnu au froment tient à sa blancheur et non à ses qualités nutritives, équivalentes, voire inférieures à celles des autres céréales. ${ }^{24}$ La préférence donnée au froment par les ecclésiastiques renvoie probablement aussi à son usage exclusif pour l'eucharistie, un besoin essentiel pour la société chrétienne. ${ }^{25}$

L'importance de la distinction entre consommation des clercs et des laïcs dans la prise en compte des destinations ne s'oppose pas à une inscription précise des diverses espèces récoltées dans le cadre des contraintes et des incitations économiques. Dans le règlement en 1171-1179 du conflit entre les paroissiens de Louvigny sur les dîmes du fief de Guesberville, les juges délégués pontificaux reconnurent à l'abbaye la possession du revenu, mais attribuèrent à l'église paroissiale une rente annuelle, d'un sextier d'orge, réservant ainsi aux religieux la dîme de la récolte

23 Harald Müller, Päpstliche Delegationgerichtsbarkeit in der Normandie (12 und frühes 13 Jahrhundert), Bonn Studien und Dokumente zur Gallia Pontificia, 4, Bonn 1997, II, no. 131, 252-3: Tota decima bladi de Fonteneto Marmion [...] deferetur ante grangiam predictorum abbatis et monachorum [scil. de Barbereio] de Fontaneto et ibi erit diuisa, sicut prius diuideratur, in platea juxta monasterium, ita quod ad decimam colligendam abbas et monachi tres quadrigas cum hominibus et equis et aliis necessariis inuenient et prefatus Robertus unam cum necessariis et sextam partem quinte quadrige cum necessariis et unius hominis gradientis per campos super equum ad decimam colligendam et custodiendam pacifice inveniet ('Toute la dîme du blé de Fontenay le Marmion sera portée devant la grange desdits abbé et moines à Fontenay et elle sera divisée comme auparavant, sur la place qui jouxte le monastère; et l'abbé et les moines devront trouver pour rassembler la dîme trois charrettes avec les hommes et tout ce qui sera nécessaire et ledit Robert, de sa bonne volonté, trouvera une autre charrette avec tout ce qui sera nécessaire, ainsi que le sixième, avec ce qui sera nécessaire, d'une cinquième charrette et d'un homme à cheval qui ira par les champs pour rassembler la dîme').

24 M. Arnoux, 'Manger ou cultiver: laboratores, oratores et bellatores entre production et consommation ( $\mathrm{XI}^{\mathrm{e}}-\mathrm{XIII}^{\mathrm{e}}$ siècle)', L'alimentazione nell'alto medioevo: pratiche, simboli, ideologie, Settimane di studio della fondazione Centro Italiano di Studi sull'Alto Medioevo, 63, Spoleto 2016, 939-62.

25 Cf. les prescriptions très précises relatives à la fabrication des hosties dans le coutumier de Lanfranc : The Monastic Constitutions of Lanfranc, éd. D. Knowles et C. N. L. Brooke, OMT, Oxford 2002, 123-7. 
de froment. Un demi-siècle plus tard, en 1230, le conflit entre les moniales de Villers-Canivet et les paroissiens de Soumont sera réglé de la même façon, les juges nommés par l'évêque de Sées donnant aux moniales la possession de la dîme et tout le froment, tandis qu'une rente annuelle, considérable, de huit sextiers d'orge et quatre d'avoine est attribuée aux paroissiens. Dans ses attendus, le jugement fait état des arguments, purement économiques, de ceux-ci, qui lient la nécessité d'affermer la dîme à son instabilité et à son caractère imprévisible (quoniam perceptiones hujusmodi [...] quasi volatiles erant et incerte), dommageable à la gestion de l'église paroissiale. Dans ce cas, comme dans le cas précédent, la sentence fait de la communauté régulière la fermière perpétuelle de la dîme, au prix d'une rente perpétuelle en céréale de printemps : la solution stabilise le revenu des paroissiens, en réservant aux religieux, plus solidement établis, le risque et les bénéfices d'une ressources irrégulière. ${ }^{26}$

Dans tous ces textes, la rigueur dans l'application des préceptes canoniques ne s'oppose donc pas à l'attention aux mécanismes économiques. Bien au contraire, dès la fin du douzième siècle, le règlement des conflits prévoit le recours à une expertise en termes marchands en cas de contestation. L'accord passé en 1181-4 entre l'abbaye de Saint-Évroult et les chanoines de Sainte-Barbe-en-Auge au sujet de l'ermitage de La Roche, précise ainsi: 'Les moines sont tenus de payer chaque année perpétuellement à nous et à notre église un muid de blé à l'octave de la fête Saint-Michel à leur grange de Norrey, à la mesure légale de ladite ville, consistant en quatre sextiers de froment, quatre d'orge et quatre d'avoine, le grain de chaque espèce estimé au prix de vente commun'. De même, en 1192, le règlement du conflit entre les chanoine de Silly-en-Gouffern et les moines de Saint-Pierre-surDives à propos de la chapelle de Maimbeville et de ses dîmes, prévoit le versement d'une rente annuelle de trente sextiers en trois céréales, à percevoir dans la grange des chanoines à Saint-Lambert, précisant que les grains versés devront être 'en blé de qualité légale estimé de prix commun, à la mesure commune et légitime de Saint-Lambert'. ${ }^{27}$

Ces précisions, qui paraissent indispensables aux rigoureux canonistes rédacteurs de ces actes, font des marchés locaux et des experts, paysans et marchands, qui y opèrent, les juges de la régularité de pratiques religieusement fondées. Elles attestent de la parfaite moralité, du point de vue de l'église, d'une évaluation monétaire des ressources alimentaire, dont la commercialisation apparaît ainsi spirituellement fondée. La centralité des institutions du marché apparaît dans cette région avec une rare évidence. La très grande diffusion des instruments de crédit fondés sur les échanges céréaliers en apporte une autre preuve. Dans un ouvrage novateur paru en 1900, l'historien du droit Paul Génestal avait montré l'ancienneté des formes de crédit pratiquées par les communautés monastiques normandes, en faisant l'hypothèse que ce flux massif de valeur monétaire en destination de la classe paysanne était la contrepartie de la quantité plus impressionnante encore de céréales que la collection des dîmes mettait à disposition des religieux, et des marchés

26 M. Arnoux, 'Remarques sur les fonctions économiques de la communauté paroissiale (Normandie, $\mathrm{XII}^{\mathrm{e}}$-XIII ${ }^{\mathrm{e}}$ siècles)', dans Liber largitorius. Etudes d'histoire médiévale offertes à Pierre Toubert par ses élèves, éd. D. Barthélemy et J.-M. Martin, Genève 2003, 426-30.

27 Müller, Päpstliche Delegationgerichtsbarkeit, II, no. 68, 173-4: Monachi teneantur inperpetuum nobis et ecclesie nostre annuatim solvere unum modium bladi in octavis Sancti Michaelis prioris apud Noerium in grangia sua ad legitimam eiusdem ville mensuram, ita uidelicet ut IIIIor sextarii sint frumenti, quatuor ordei, IIIIor avene, cuius bladi singule species communis uenditionis pretio debeant estimari, et no. 89, 196-7: in blado legali et pretio communi estimabili ad communem et legittimam mensuram Sancti Lamberti. 
urbains sur lesquels ces derniers étaient actifs. Il mettait en évidence le rôle joué par les canonistes pontificaux dans le contrôle et la modification progressive de pratiques économiques associant aussi bien clercs et laïcs que les laïcs entre eux. ${ }^{28}$

À partir des premières années du treizième siècle, l'apparition d'instruments de crédit stipulés à la fois en mesures de grain et en monnaie métallique apporte la preuve que les marchés céréaliers établis dans les campagnes sont assez abondants, équilibrés et prévisible pour que s'établisse une circulation de valeur monétaire gagée par l'arrivée annuelle des récoltes sur les marchés à l'automne. Un contrat de 1209 liant les religieuses de Saint-Amand de Rouen aux moines de BonneNouvelle autour d'un versement annuel considérable de quatre muids de froment à verser sur le marché aux grains du vendredi à Rouen est le premier indice du fonctionnement de ce système. ${ }^{29}$ À partir des années 1220 , on voit des contrats de ce genre se répandre dans les campagnes, impliquant pour l'essentiel des communautés religieuses et des paysans, autour d'un marché unique. C'est le cas autour de Caen, où les moines de Savigny acquièrent ainsi par dizaines des rentes payables en froment, orge, ou avoine sur le marché de Thaon, tandis que ceux de Troarn font de même sur le marché de Touffréville, et ceux de Saint-Étienne de Fontenay sur les marchés de Fontenay, Cingal et Thury. Il s'agit d'un dossier appelé à s'étoffer, et qui fournira des informations de grande importance sur les modes de fonctionnement des marchés normands. ${ }^{30}$

Dans l'optique de notre recherche, ces multiples documents, mis en regard des sources flamandes et rhénanes (mais aussi italiennes) qui donnent une importance majeure aux institutions urbaines, ou des sources anglaises, qui placent au centre du dispositif économique les institutions seigneuriales enracinées dans les paysages agraires, suggèrent que les marchés ont en Normandie un rôle central dans l'organisation de l'accès aux richesses nécessaires. C'est par leur intermédiaire que peut se construire une notion de ressource qui soit fidèle au contenu des sources, et peut-être aussi aux pratiques des acteurs. Les systèmes de ressources qui peuvent être ainsi mis en évidence sont clairement différents selon les régions, mais rien ne permet d'affirmer que les possibilités offertes par l'environnement suffisent à expliquer ces variations régionales. Les sources médiévales, lorsqu'on les interroge dans cette perspective, attirent plutôt l'attention sur l'importance des institutions religieuses et des choix et politiques, aussi bien dans les dynamiques de convergences entre régions que dans l'émergence de caractères originaux.

\footnotetext{
28 R. Génestal, Le rôle des monastères comme établissements de crédit, étudié en Normandie du xie siècle à la fin du xiiie siècle, Paris 1901.

29 I. Theiller, 'Prix du marché, marché du grain et crédit au début du XIII ${ }^{\mathrm{e}}$ siècle : autour d'un dossier rouennais', Le Moyen Age, 2009, 252-76.

30 Je prépare une publication sur ce sujet.
} 